2016 Spring Technical Meeting

Central States Section of the Combustion Institute

May 15-17, 2016

Knoxville, Tennessee

\title{
A Burke-Schumann Analysis of Dual-Flame Structure Supported by a Burning Droplet
}

\author{
V. Nayagam ${ }^{1}$, D. Dietrich ${ }^{2}$, and F. A. Williams ${ }^{3}$ \\ ${ }^{1}$ Department of Mechanical and Aerospace Engineering \\ Case Western Reserve University, Cleveland, OH 44106, USA \\ ${ }^{2}$ NASA Glenn Research Center, Cleveland, OH 44135,USA \\ ${ }^{3}$ Department of Mechanical and Aerospace Engineering \\ University of California San Diego, La Jolla, CA 92093, USA
}

\begin{abstract}
Droplet combustion experiments carried out onboard the International Space Station (ISS), using pure fuels and fuel mixtures, have shown that quasi-steady burning can be sustained by a non-traditional flame configuration, namely a "cool flame" burning in the "partial-burning" regime where both fuel and oxygen leak through the low-temperaturecontrolled flame-sheet. Recent experiments involving large, bi-component fuel (n-decane and hexanol, 50/50 by volume) droplets at elevated pressures show that the visible, hotflame becomes extremely weak while the burning rate remains relatively high, suggesting the possibility of simultaneous presence of "cool" and "hot" flames of roughly equal importance. The radiant output from these bi-component droplets is relatively high and cannot be accounted for only by the presence of a visible hot-flame. In this analysis we explore the theoretical possibility of a dual-flame structure, where one flame lies close to the droplet surface called the "cool-flame," and other farther away from the droplet surface, termed the "hot-flame." A Burke-Schumann analysis of this dual-structure seems to indicate such flame structures are possible over a narrow range of initial conditions. Theoretical results can be compared against available experimental data for pure and bi-component fuel droplet combustion to test how realistic the model may be.
\end{abstract}

Keywords: Dual flames, cool flames, droplet combustion

\section{Introduction}

Droplet combustion experiments conducted in space onboard the International Space Station with n-alkane fuels showed that quasi-steady combustion can be sustained with the regular hotflame chemistry or with the low-temperature, cool-flame chemistry controlled by the negative temperature coefficient (NTC) region $[1,2,3]$. The cool flames were found to lie closer to the droplet surface, with the flame standoff ratios around three, and the flame temperatures were around $780 \mathrm{~K}$, corresponding the NTC-region temperature for n-alkanes $[3,2,4]$. On the other hand, the traditional hot-flames were located farther away [5] and their flame temperatures were the adiabatic flame temperatures determined by the fuel thermodynamic properties. In all these studies, a large droplet when ignited in a quiescent microgravity environment, burns initially with visible hot flame, and then extinguishes radiatively as the flame size grows at a finite droplet diameter. Subsequently, a cool flame supported quasi-steady combustion begins without a visible 
flame which also extinguished at a finite droplet diameter [6]. Because of this behavior coolflame combustion has also been called "second-stage" combustion [3,6].

More recent space experiments with n-decane and hexanol mixtures (50/50 by volume) at elevated pressures show some intriguing results [7]. It was found that the hot-flame luminosity from large droplets that extinguished radiatively, measured with a color camera as well as a low-light level ultraviolet camera (LLUV) with a spectral filter centered at $310 \mathrm{~nm}$ that captured the $O H^{*}$ chemiluminescence, continually decreased when the ambient pressure was increased from 0.05 $\mathrm{MPa}$ to $0.3 \mathrm{MPa}$. However, the flame radiance during hot-flame combustion, measured using a broadband radiometer, remained almost a constant regardless of the ambient pressure. It was also observed that the cool flames that formed immediately following hot-flame extinction had radiant outputs that increased with pressure, reaching almost one-half of the hot-flame radiance at $0.3 \mathrm{MPa}$. These observations lead these authors to speculate the possibility of simultaneous presence of cool and hot flames prior to complete extinction of hot flames at higher pressures $[7]$.

The objective of the present study is to explore the possibility of such dual-flame structures during quasi-steady droplet combustion using the Burke-Schumann analysis where it is assumed that the reactions are confined to a thin flame sheet. Clearly, for the dual flames to exist the gasphase chemistry should proceed in two distinct steps with each step having a distinct activation energy that is relatively high. In the premixed flame context, Kapila and Ludford [8] considered a sequential reaction of the form $A \rightarrow B \rightarrow C$, and showed two distinct, separated flames are possible under certain conditions using activation energy asymptotics. Normal alkane chemistry, which is of interest here, is much more complex [9]. However, in a recent study [10] it has been shown that for dimethyl ether (DME), which exhibits NTC behavior, a separated cool-flame front and a hot-flame front can be present simultaneously when the mixture is ignited by a hot pocket. We consider here a much simpler configuration where a cool flame is located closer to the droplet surface and a hot flame farther away, with the cool flame acting as a fuel-rich premixed flame leaking a substantial amount of fuel through the flame, while the weak hot flame acts as a fuellean premixed flame leaking oxygen to feed the cool flame. Though we assume that fuel and oxygen are the reactants in both flames, the stoichiometry and heat of combustion are treated as independently specifiable variables for both the reactions. To the best of our knowledge similar analyses for diffusion flames are not available in the literature. The problem formulation is described in the next section, followed by solutions and discussion of the results.

\section{Formulation}

Consider a fuel droplet burning quasi-steadily in a microgravity environment under spherically symmetric conditions as illustrated in Fig. 1. The burning droplet supports two diffusion flames, one closer to the droplet surface with fuel leakage, termed the "cool flame" here for convenience, and the other farther away, called the "hot flame", where the remaining fuel is consumed while oxygen is only partially consumed. It is assumed that the reactions are confined to a thin flamesheet and frozen everywhere else. A general one-step, irreversible chemistry of the following form is assumed for the cool and hot flames, namely

$$
\begin{aligned}
& \left.\left.1 \mathrm{~kg}[\text { Fuel }]+\nu_{c} \mathrm{~kg} \text { [Oxidizer }\right] \rightarrow\left(1+\nu_{c}\right) \mathrm{kg} \text { [Products1] }+q_{c} \text { [Heat }\right], \\
& \left.1 \mathrm{~kg}[\text { Fuel }]+\nu_{h} \mathrm{~kg} \text { [Oxidizer] } \rightarrow\left(1+\nu_{h}\right) \mathrm{kg} \text { [Products2] }+q_{h} \text { [Heat }\right]
\end{aligned}
$$






Figure 1: Schematic illustration of dual-flame structure surrounding a droplet with leakage of fuel in the inner "cool" flame, and oxygen through the outer "hot" flame.

where $\nu_{i}$ and $q_{i}$ are the stoichiometric coefficients and heats of combustion per unit mass of fuel consumed, with the subscript $c$ denoting the cool flames and $h$ the hot flames. The conservation equations for gas phase mass, energy, and species without the source terms can be written as, Mass:

$$
\frac{1}{r^{2}} \frac{d}{d r}\left(r^{2} \rho u\right)=0
$$

Energy:

$$
u \frac{d T}{d r}-\frac{\rho D}{\rho r^{2}} \frac{d}{d r}\left(r^{2} \frac{d T}{d r}\right)=0
$$

Species:

$$
u \frac{d Y_{i}}{d r}-\frac{\rho D}{\rho r^{2}} \frac{d}{d r}\left(r^{2} \frac{d Y_{i}}{d r}\right)=0, \quad i=F, O
$$

where $u$ is the radial velocity, $r$ is the radial coordinate, $Y_{i}$ is the mass fraction of species $i, T$ is the temperature, $\rho$ is the density, and $D$ is the mass diffusivity. The subscripts $i=F$, and $O$ denote fuel, and oxygen, respectively, and we have assumed unity Lewis number $\left(\rho D=\lambda / c_{P}\right)$ where $\lambda$ is the thermal conductivity, and $c_{p}$ is the specific heat of the gas.

The mass conservation equation (3) simply states that the mass flow through any spherical shell is constant, namely $\dot{m}=4 \pi r^{2} \rho u$, is a constant. A new independent variable $\eta$ can then be defined as

$$
\eta=1-e^{-\dot{M} / r},
$$

where $\dot{M}=\dot{m} /(4 \pi \rho D)=r^{2} \rho u /(\rho D)$, which is a constant. The conservation equations for species and energy can be expressed in terms of dimensionless dependent variables $\tilde{Y}_{O}=Y_{O} / \nu_{h}$, $\tilde{Y}_{F}=Y_{F}$, and $\tilde{T}=c_{P} T / q_{h}$ as

$$
\frac{d^{2} \tilde{Y}_{F}}{d \eta^{2}}=\frac{d^{2} \tilde{Y}_{O}}{d \eta^{2}}=\frac{d^{2} \tilde{T}}{d \eta^{2}}=0
$$


The semi-infinite domain $0<r<\infty$ is mapped into $1>\eta>0$. Note that the dependent variables are linear functions of the independent variable $\eta$. A schematic illustration of the species and temperature profiles in $\eta$ coordinate are shown in Fig. 2. The boundary conditions associated with Eq. (7) at the fuel surface $\left(r=r_{s}\right.$ or $\left.\eta=\eta_{s}\right)$ and in the ambient environment $(r \rightarrow \infty$ or $\eta=0)$ are

$$
\begin{aligned}
& \eta=\eta_{s}: \quad \tilde{T}=\tilde{T}_{s} \\
& \left.\frac{d \tilde{T}}{d \eta}\right|_{\eta_{s}}=-\frac{\tilde{L}}{1-\eta_{s}} \\
& \left.\frac{d \tilde{Y}_{F}}{d \eta}\right|_{\eta_{s}}=\frac{1-\tilde{Y}_{F s}}{1-\eta_{s}} \\
& \eta=0: \quad \tilde{Y}_{F}=0, \quad \tilde{Y}_{O}=\tilde{Y}_{O \infty}, \quad \tilde{T}=\tilde{T}_{\infty}
\end{aligned}
$$

In the classical Burke-Schumann analysis, it is assumed that the fuel and oxygen mass fractions at the flame-sheet are zero, and they meet at stoichiometric proportions. However, in the dualflame configuration considered here, we allow for the fuel to leak through the cold-flame and oxygen through the hot-flame. The leakage fractions $a$ and $b$, for fuel and oxygen, respectively, are treated as variable parameters. The slopes of fuel and oxygen profiles across the flame-sheets are then related according to

$$
\left.\frac{d \tilde{Y}_{F}}{d \eta}\right|_{\eta_{c}^{-}}=\left.a \frac{d \tilde{Y}_{F}}{d \eta}\right|_{\eta_{c}^{+}} ;\left.\quad \frac{d \tilde{Y}_{O}}{d \eta}\right|_{\eta_{h}^{+}}=\left.b \frac{d \tilde{Y}_{O}}{d \eta}\right|_{\eta_{h}^{-}},
$$

where $\eta_{h}$ and $\eta_{c}$ are the hot and cold-flame locations (see, Fig. 2). The stoichiometric requirements at the hot and the cold flame-sheets, using Eqs. (8) and (10), are

$$
\begin{aligned}
& \left.\frac{d \tilde{Y}_{O}}{d \eta}\right|_{\eta_{c}^{-}}=-(1-a) s \frac{1-\tilde{Y}_{F s}}{1-\eta_{s}} \\
& \left.\frac{d \tilde{Y}_{O}}{d \eta}\right|_{\eta_{h}^{-}}=-\frac{a}{(1-b)} \frac{1-\tilde{Y}_{F s}}{1-\eta_{s}}
\end{aligned}
$$

where $s=\nu_{c} / \nu_{h}$. In addition, the jump conditions for temperature across the flame-sheets due to heat release are

$$
\begin{aligned}
-\left.\frac{d \tilde{Y}_{F}}{d \eta}\right|_{\eta_{h}^{+}} & =\left.\frac{d \tilde{T}}{d \eta}\right|_{\eta_{h}+}-\left.\frac{d \tilde{T}}{d \eta}\right|_{\eta_{h}^{-}} \\
-\left.(1-a) \frac{d \tilde{Y}_{F}}{d \eta}\right|_{\eta_{c}^{+}} & =\frac{1}{\tilde{q}}\left(\left.\frac{d \tilde{T}}{d \eta}\right|_{\eta_{c}+}-\left.\frac{d \tilde{T}}{d \eta}\right|_{\eta_{c}^{-}}\right)
\end{aligned}
$$

where $\tilde{q}=q_{c} / q_{h}$. Since the species and temperature profiles are piecewise-linear in $\eta$ coordinate, the slopes appearing in Eqs. $(7,10-12)$ can be directly related to the unknown parameters of the problem, namely, $\tilde{Y}_{F s}, \eta_{s}, \tilde{T}_{c}, \tilde{Y}_{F c}, \eta_{c}, \tilde{T}_{h}, \tilde{Y}_{O h}$, and $\eta_{h}$. The resulting eight nonlinear algebraic equations for the eight unknowns are

$$
\frac{\tilde{Y}_{F s}-\tilde{Y}_{F c}}{\eta_{s}-\eta_{c}}=\frac{1-\tilde{Y}_{F s}}{1-\eta_{s}}
$$




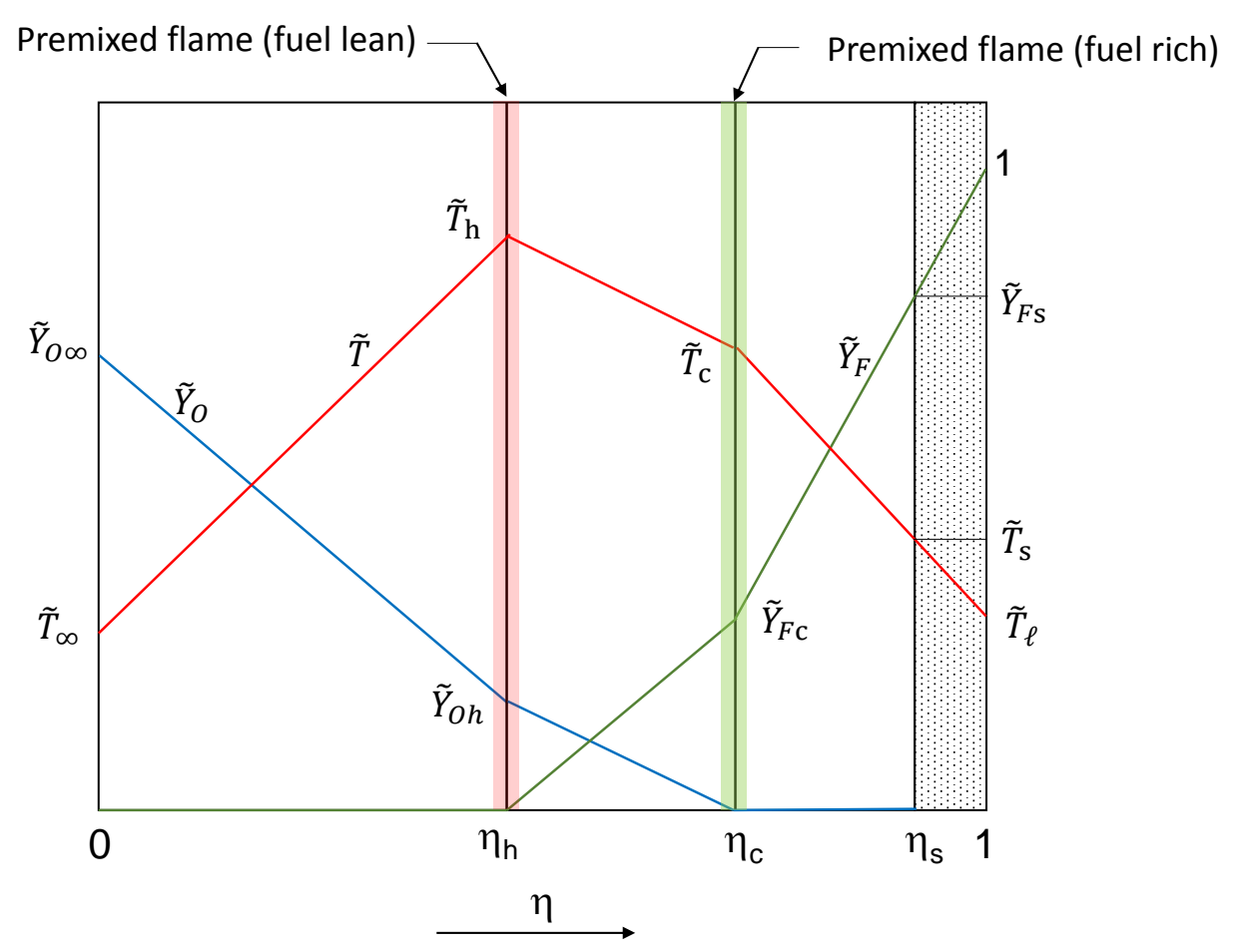

Figure 2: Dual-flame profiles in $\eta$ coordinate.

$$
\begin{gathered}
\frac{\tilde{T}_{s}-\tilde{T}_{c}}{\eta_{s}-\eta_{c}}=-\frac{\tilde{L}}{1-\eta_{s}} \\
\frac{\tilde{Y}_{F c}}{\eta_{c}-\eta_{h}}=a \frac{1-\tilde{Y}_{F s}}{1-\eta_{s}} \\
\frac{\tilde{Y}_{O h}}{\eta_{c}-\eta_{h}}=-b \frac{\tilde{Y}_{O h}-\tilde{Y}_{O \infty}}{\eta_{h}} \\
\frac{\tilde{Y}_{O h}}{\eta_{c}-\eta_{h}}=(1-a) s \frac{1-\tilde{Y}_{F s}}{1-\eta_{s}} \\
\frac{\tilde{Y}_{O h}-\tilde{Y}_{O \infty}}{\eta_{h}}=-\frac{a}{(1-b)} \frac{1-\tilde{Y}_{F s}}{1-\eta_{s}} \\
\frac{\tilde{T}_{s}-\tilde{T}_{c}}{\eta_{s}-\eta_{c}}-\frac{\tilde{T}_{c}-\tilde{T}_{h}}{\eta_{c}-\eta_{h}}=-(1-a) \tilde{q} \frac{1-\tilde{Y}_{F s}}{1-\eta_{s}} \\
\frac{\tilde{T}_{c}-\tilde{T}_{h}}{\eta_{c}-\eta_{h}}-\frac{\tilde{T}_{h}-\tilde{T}_{\infty}}{\eta_{h}}=-\frac{\tilde{Y}_{F c}}{\eta_{c}-\eta_{h}}
\end{gathered}
$$

Equations (13-20) are solved symbolically using the Solve procedure in Mathematica [11] to obtain analytical results. The accuracy of these results are checked by solving the same equations numerically and comparing. The analytical solutions were also tested by considering the limiting conditions for which the results are well known. 


\section{Solution Procedure}

Explicit solutions to the set of nonlinear algebraic equations (13-20) are obtained by treating $a, s, q, \tilde{Y}_{O \infty}, \tilde{T}_{\infty}$, and $\eta_{s}$ as known parameters. By treating $\eta_{s}$ as a known parameter we are essentially specifying the burning rate and calculating the resulting flame configurations. It is worthwhile to note that a number of results can be obtained rather easily by inspection. Since we have forced the fuel and oxygen to be completely consumed between the two flames, $a$ and $b$ cannot be independently specified when the stoichiometric ratio $s$ is fixed. It is easy to show using Eqs. (16-18) that

$$
s=\frac{a b}{(1-a)(1-b)} .
$$

When $a$ is zero all the fuel is consumed at the cool flame and $b$ becomes one showing that the hot flame ceases to exist since no oxygen is consumed. The reverse happens when $a$ is unity and $b$ becomes zero. From Eqs. (15) and (17) it is also seen that

$$
\frac{\tilde{Y}_{O h}}{\tilde{Y}_{F c}}=\frac{(1-a) s}{a},
$$

showing $\tilde{Y}_{F c}$ and $\tilde{Y}_{O h}$ are related by a constant irrespective of the burning rate. The solutions to equations (13-20) can be further simplified by first considering the three boundary conditions at the fuel surface first. From equations (13) and (14) the following two identities, showing continuity of slopes across the droplet surface, can be derived, namely,

$$
\frac{1-\tilde{Y}_{F s}}{1-\eta_{s}}=\frac{1-\tilde{Y}_{F c}}{1-\eta_{c}} ; \quad \frac{\tilde{T}_{s}-\tilde{T}_{c}}{\eta_{s}-\eta_{c}}=\frac{\tilde{T}_{\ell}-\tilde{T}_{c}}{1-\eta_{c}},
$$

where we have defined $\tilde{T}_{\ell}=\left(\tilde{T}_{s}-\tilde{L}\right)$. Using these identities $\tilde{Y}_{F s}, \tilde{T}_{s}$, and $\eta_{s}$ can be eliminated from equations (15) through (20) and the resulting set of six equations can be solved, now treating $\eta_{c}$ as one of the independent variables. In addition, the species profiles are independent of the temperature profiles, and they they can be solved first and then used to compute the temperature profile conditions expressed in equation (19) and (20), if desired. The dimensionless droplet-surface location $\eta_{s}$ is related to the cool-flame location $\eta_{c}$ as

$$
\left(1-\eta_{s}\right)=-\frac{\tilde{L}}{\left(\tilde{T}_{\ell}-\tilde{T}_{c}\right)}\left(1-\eta_{c}\right)
$$

The mass burning rate of the droplet $\dot{m}$, and the burning rate constant $K=4 d\left(r_{s}^{2}\right) / d t$ are related to $\eta_{s}$ as

$$
\dot{m}=4 \pi \rho D r_{s} \ln \left(\frac{1}{1-\eta_{s}}\right) ; \quad K=\frac{8 \rho D}{\rho_{\ell}} \ln \left(\frac{1}{1-\eta_{s}}\right) .
$$

The hot and cool flame standoff ratios, $f_{h}=\left(r_{h} / r_{s}\right)$ and $f_{c}=\left(r_{c} / r_{s}\right)$, respectively, can be expressed as

$$
f_{h}=\ln \left(\frac{1-\eta_{s}}{1-\eta_{h}}\right) ; \quad f_{c}=\ln \left(\frac{1-\eta_{s}}{1-\eta_{c}}\right) \text {. }
$$




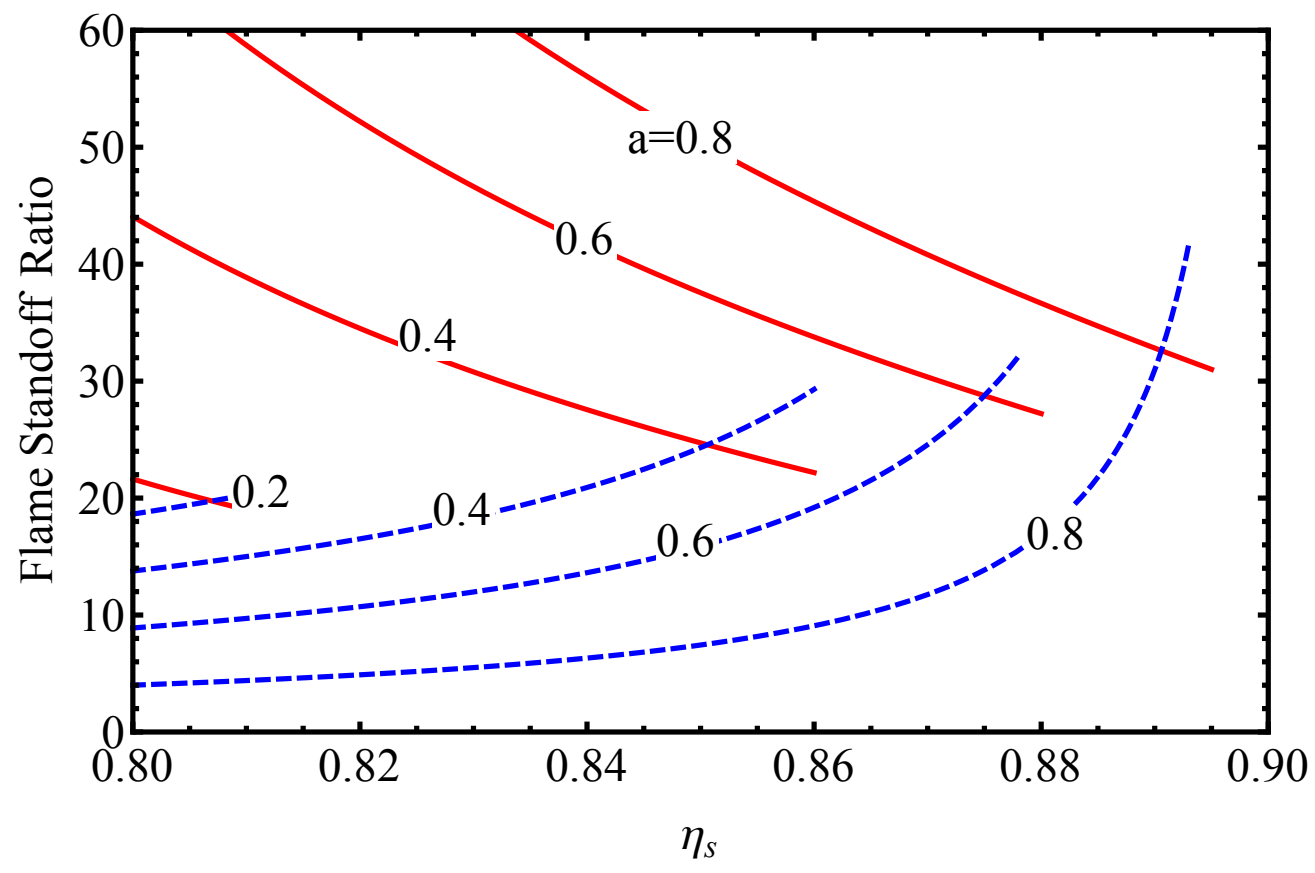

Figure 3: Hot-flame (solid line) and cool-flame (dashed line) standoff ratios for various values of $a$ as a function of $\eta_{s}$ and $\tilde{q}=0.2$.

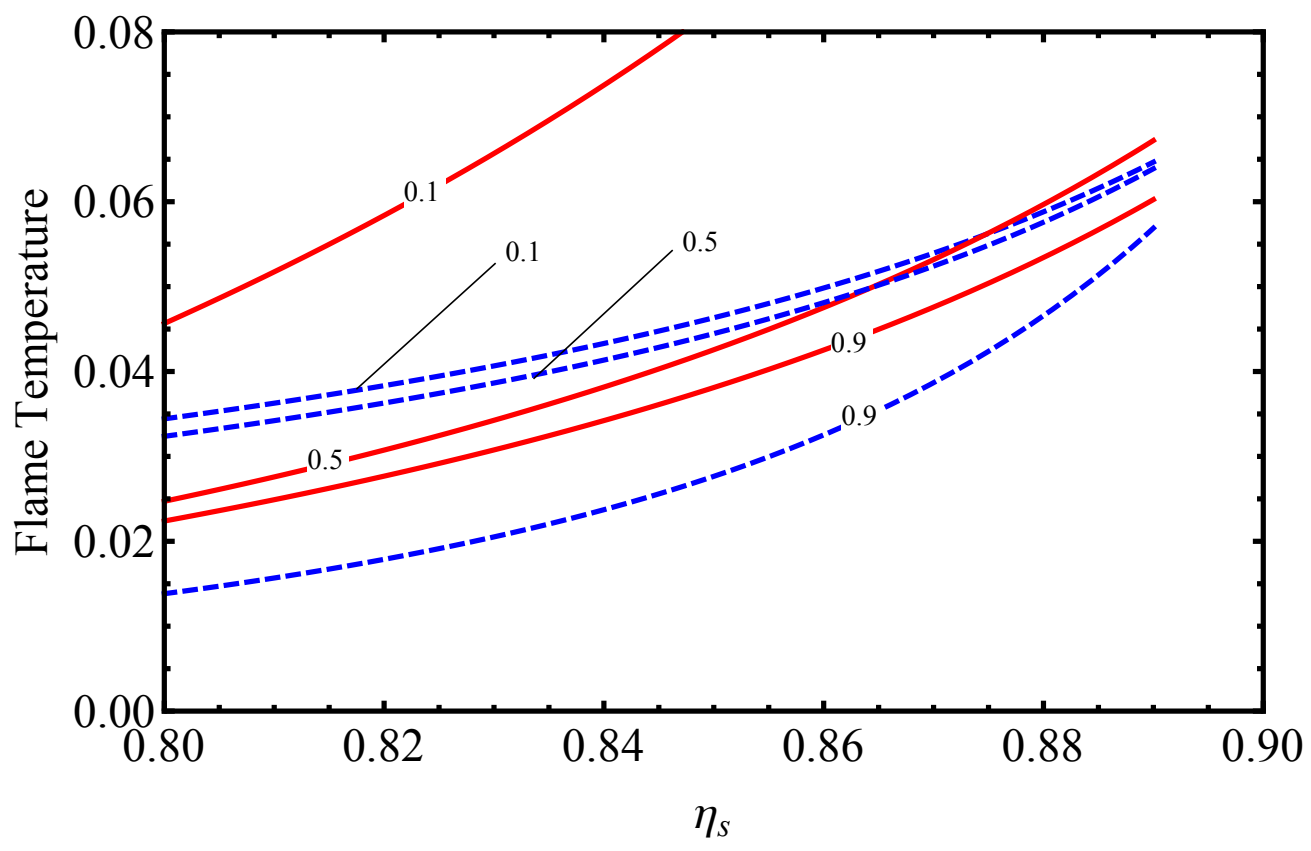

Figure 4: Hot-flame (solid line) and cool-flame (dashed line) temperatures for various values of $a$ as a function of $\eta_{s}$ and $\tilde{q}=0.2$. 


\section{Results and Discussions}

The analytical solutions obtained using Mathematica are cumbersome and lengthy. We present here the key aspects of the results in graphical form, keeping most of the independently specifiable parameters fixed. Since one of our objective is to understand the influences of underlying chemical kinetics on the dual-flame structures, we vary the parameters related to chemistry, namely the amount of fuel that leaks through the cool flame $a$, and the ratio of heat of combustion for the cool flame and the hot flame per unit mass of fuel consumed, $\tilde{q}$. A more thorough parametric study of the results will be presented elsewhere [12]. The values of independent nondimensional parameters used in the calculations, roughly corresponding to n-alkanes burning in air, are given in Table 1 . Assuming $\rho / \rho_{\ell}$ is of the order $1.5 \times 10^{-3}$, and the value of $D$ approximately $0.1 \mathrm{~cm}^{2} / \mathrm{s}$, it can be shown using Eq. (25) that when the burning rate constant $K$ varies between 0.3 to $.7 \mathrm{~mm}^{2} / \mathrm{s}$, the dimensionless droplet surface location $\eta_{s}$ varies between 0.7 and 0.95. Furthermore, based on experimental observations, we focus here on conditions under which the cool flame is located closer to the droplet surface, namely $\eta_{h}<\eta_{c}<\eta_{s}$.

Table 1: Values of the nondimensional parameters used in the calculations.

\begin{tabular}{lc}
\hline \hline Variable & Value \\
\hline & \\
Heat of vaporization, $\tilde{L}$ & $7 \times 10^{-3}$ \\
Ambient oxygen concentration, $\tilde{Y}_{O \infty}$ & 0.066 \\
Ambient temperature, $\tilde{T}_{\infty}$ & .007 \\
Droplet surface temperature, $\tilde{T}_{s}$ & .009 \\
Stoichiometric coefficient ratio, $s$ & 0.7 \\
Heat of combustion ratio, $\tilde{q}$ & $0.01-0.3$ \\
Fuel leakage fraction, $a$ & $0.0-1.0$ \\
Burning rate in terms of $\eta_{s}$ & $0.8-0.9$ \\
\hline
\end{tabular}

Figure 3 shows the variations of cool and hot flame standoff ratios, $f_{h}$ and $f_{c}$, as a function of $\eta_{s}$ for various values of fuel leakage fraction $a$ for a constant value of $\tilde{q}=0.2$. As $a$ increases the fuel leakage at the cool flame increases, and correspondingly the oxygen leakage at the hot flame decreases. For a fixed value of $\eta_{s}$, the cool flame moves closer to the fuel surface with increasing $a$ in order to maintain the required heat flux to the droplet surface. Since more fuel is now available to the hot flame, it moves farther away from the droplet surface to meet the stoichiometric requirement. For a fixed value of $a$, as the burning rate is increased the cool flame moves farther away due to the increased surface blowing velocity. However, the hot flame moves closer to the cool flame supplying added heat to it and thus increasing the overall heat transfer to the fuel surface causing increased vaporization. At some point the hot and cool flames merge together and there are no physically meaningful results beyond that burning rate.

Figure 4 shows the cool flame temperature $\tilde{T}_{c}$, and the hot flame temperature $\tilde{T}_{h}$ plotted as function of $\eta_{s}$ for various values of $a$, holding $\tilde{q}=0.2$. Both flame temperatures increase as the burning rate increases due to the increased amount of fuel being burnt. For a fixed value of $\eta_{s}$, both the flame temperatures decrease with increasing fuel leakage at the cool flame. However, the rate of decrease for each of the flame is quite different: initially as $a$ increase from 0 to approximately 


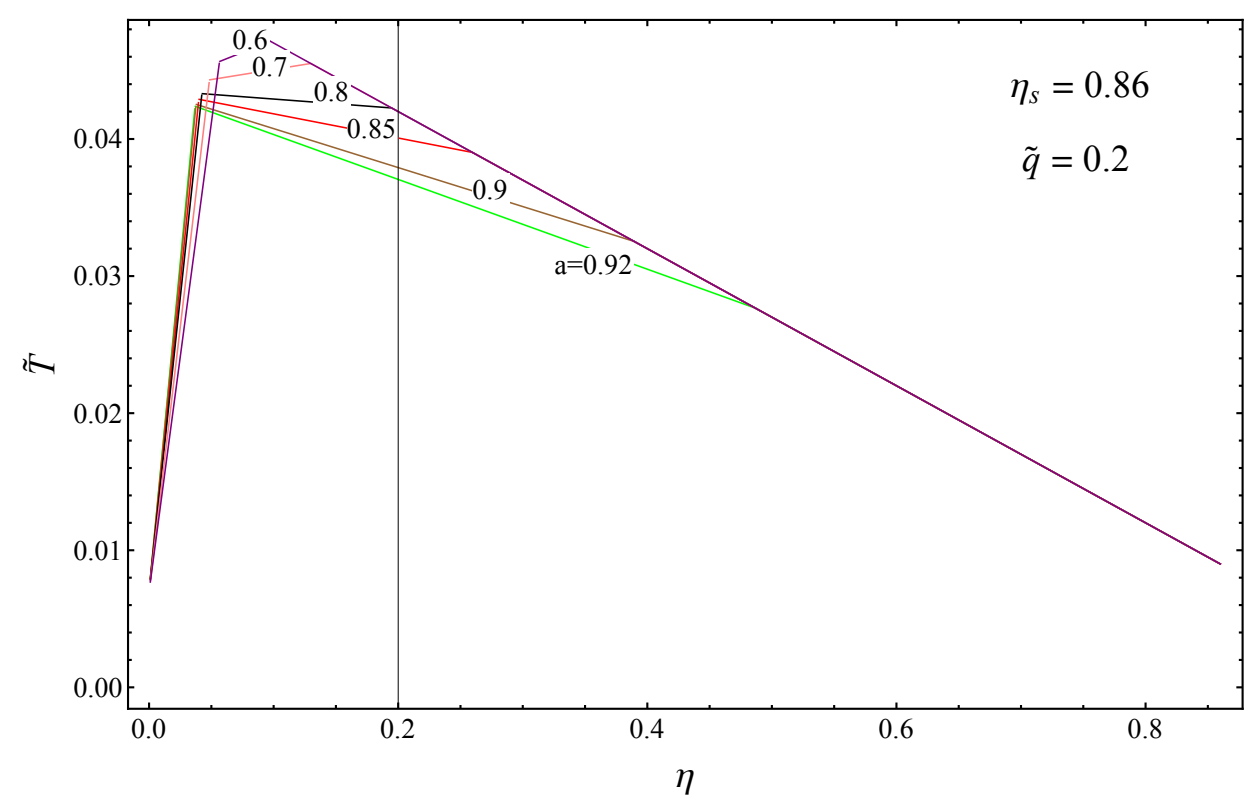

Figure 5: Dimensionless temperature profiles for varying fuel leakage fraction $a: \eta_{s}=0.86$, $\tilde{q}=0.2$.

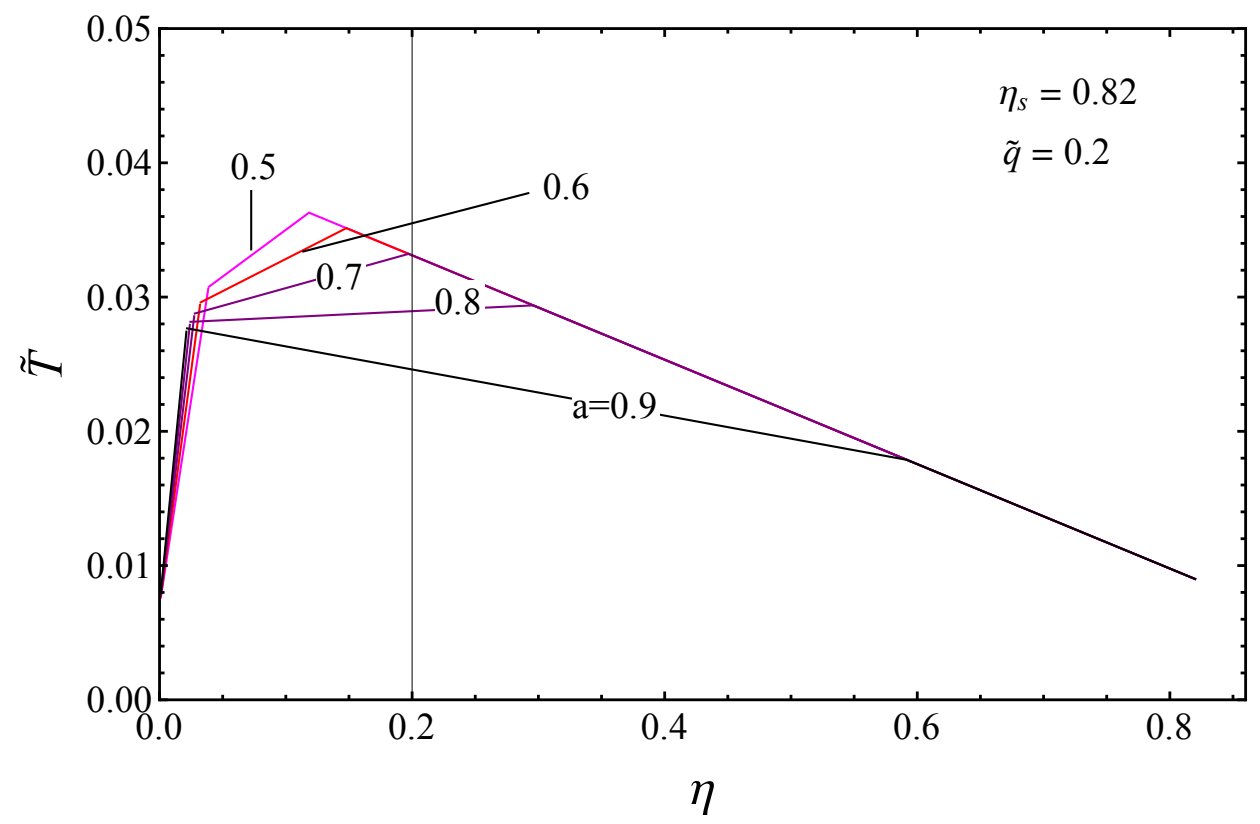

Figure 6: Dimensionless temperature profiles for varying fuel leakage fraction $a: \eta_{s}=0.82$, $\tilde{q}=0.2$. 




Figure 7: Dimensionless temperature profiles for varying heat of reaction fraction $\tilde{q}: \eta_{s}=0.86$, $a=0.9$.



Figure 8: Dimensionless temperature profiles for varying heat of reaction fraction $\tilde{q}: \eta_{s}=0.82$, $a=0.5$. 
0.5, the hot flame temperature decreases much faster while the cold flame temperature remains relatively fixed, and the reverse happens when $a$ is increased further. When $a$ is around 0.5 , the hot flame temperature actually falls below the cool flame temperature at lower burning rates. When $a$ is increased further $\tilde{T}_{c}$ begins to fall at a faster rate and becomes lower than $\tilde{T}_{h}$. Increasing $a$ for a fixed burning rate causes the flames to move further apart and reduces the flame temperatures to maintain the overall energy conservation.

Figure 5 illustrates the the overall flame structure in $\eta$ coordinate for a burning rate corresponding to $\eta_{s}=0.86$ and $\tilde{q}=0.2$ for seven different values of $a$. As the leakage fraction increases in the cool flame, its flame temperature decreases. However, to keep the burning rate constant it moves closer to the droplet surface. The hot flame, on the other hand, moves farther away with increasing fuel leakage to capture a sufficient amount of oxygen and its temperature decreases slightly as described before. It is interesting to note that as $a$ approaches one, the cool flame moves closer to the droplet surface and eventually coincides with it so only a single hot flame exists in the gas phase supplying most of the heat. When $a$ approaches zero, both the flames move closer to each other and merge eventually, with the cool flame supplying all the heat. A similar scenario is shown in Fig. 6 for slightly lower burning rate, $\eta_{s}=0.82$. For this lower burning rate, the leakage fraction at which both the flame temperature are the same occurs around $a=0.8$. On the other hand, when $\eta_{s}=0.86$, the hot flame still supplies heat to the cool flame when $a=0.8$.

Now we consider the effects of varying the heat combustion of the cool flame $\tilde{q}$. We consider two different cases; first a relatively weak cool flame with $a=0.9$ shown in Fig. 7 , and a robust cool flame with $a=0.5$ in Fig. 8. In the weak cool-flame case as the heat of reaction decreases, the cool flame moves closer to the droplet and eventually loses its identity as $\tilde{q}$ approaches zero, leaving a single hot flame. It should also be noted that as $\tilde{q}$ increases the amount of heat the cool flame receives from the hot flame decreases, shown by the decrease in slope of the temperature profile between $\eta_{h}$ and $\eta_{c}$. The more robust cool-flame case, shown Fig. 8, has cool-flame temperatures are greater than that of the hot-flame. As the heat of combustion ratio approaches zero, the cool flame moves closer to the hot flame and eventually a single flame is established. It is also interesting to note that a dominant cool flame, i.e, $\tilde{T}_{c}>\tilde{T}_{h}$, is established with the fuel leakage of only $50 \%$ and relative heat of combustion of only $10 \%$.

\section{Concluding Remarks}

The analysis presented here shows that dual flames during spherically symmetric droplet combustion are possible when fuel leakage occurs under certain conditions. With the heat of combustion of the cool flame around $20 \%$ of the hot flame, a weak cool flame is established when the fuel leakage is high. The weak flame moves toward the droplet surface, as its heat combustion increases. One the other hand, a relatively robust coll flame with fuel leakage fraction less than $50 \%$ behaves differently; as the heat of combustion is decreased the cool flame moves closer to the hot flame and eventually merges with forming a single flame. More detailed analyses of these results are currently underway. 


\section{Acknowledgments}

This work was supported by the NASA Space Life and Physical Sciences Research and Applications Program, and the International Space Station Program. Mr. J. Mark Hickman served as the project manager.

\section{References}

[1] V. Nayagam, D.L. Dietrich, P.V. Ferkul, M.C. Hicks, and F.A. Williams. Can cool flames support quasi-steady alkane droplet burning? Combustion and Flame, 159(12):3583 3588, 2012.

[2] A. Cuoci, A. Frassoldati, T. Faravelli, and E. Ranzi. Cool flames in droplet combustion. XXXVI Meeting of the Italian Section of the Combustion Institute, 2013.

[3] T.I. Farouk and F.L. Dryer. Isolated n-heptane droplet combustion in microgravity: "cool flames" two-stage combustion. Combustion and Flame, 161(2):565 - 581, 2014.

[4] V. Nayagam, D. L. Dietrich, and F. A. Williams. Partial-burning regime for quasi-steady droplet combustion supported by cool flames. AIAA Journal, doi: 10.2514/1.J054437, 2016, (in press).

[5] Y.C. Liu, C.T. Avedisian, K.N. Trenou, J.K. Rah, and M.C. Hicks. Experimental study of initial diameter effects on convection-free droplet combustion in the standard atmosphere for n-heptane, n-octane, and n-decane: International space station and ground-based experiments (AIAA 2014-1019). 52nd Aerospace Sciences Meeting. January, 2014.

[6] V. Nayagam, D. L. Dietrich, M. C. Hicks, and F. A. Williams. Cool-flame extinction during n-alkane droplet combustion in microgravity. Combustion and Flame, 162(5):2140-2147, 2015.

[7] D.L. Dietrich, R. Calabria, P. Massoli, V. Nayagam, and F.A. Williams. Experimental observations of the low-temperature burning of decane/hexanol droplets in microgravity. Combustion Science and Technology, (submitted, 2016).

[8] A. K. Kapila and G.S.S.Lundford. Two-step sequential reactions for large activation energies. Combustion and Flame, 29:167-176, 1977.

[9] C.K.Westbrook, W.J.Pitz, O. Herbinet, H. J.Curran, and E.J. Silke. A comprehensive detailed chemical kinetic reaction mechanism for combustion of n-alkane hydrocarbons from n-octane to n-hexadecane. Combustion and Flame, 156(1):181-199, 2009.

[10] P. Zhao, W. Liang, S. Deng, and C. K. Law. Initiation and propagation of laminar premixed cool flames. Fuel, 166:477-487, 2016.

[11] Mathematica 10.3. Wolfram Research, Inc, Champaign, Illinois, 2015.

[12] D.L. Dietrich, V. Nayagam, and F.A. Williams. A burke-schumann analysis of dual-flame structure supported by a burning droplet. (in preparation, 2016). 